\title{
El mapeo de las necesidades como medio de mediación social
}

\section{Ada Manfreda}

Università del Salento (Italia)

<info@adamanfreda.com>

Egiatan entzutea beharrekoa da bitartekoaren eginkizuna betetzen denean, eta kontsumitzaile izateaz gain, ekoizle gisara ere ulertu behar da erabiltzailea. Horretarako, derrigorrekoa da bideak izan eta sistema egokiez jabetzea erabiltzailearen esana entzun eta jasotzearren. Hori lortzeko baliabidea da beharrak mapan lekutzea. Beharrak mapan lekutzea ikerketarako metodologia izateaz gain, pedagogia eta hezkuntza-mailako eskuhartzea ere bada, hau da, subjektuaren beharrak agerrarazten saiatzen da prozesu horren bidez, eta forma ematen dio errealitatearen dimentsiora eramanez, gauzagarri bilakatuz eta, horren ondorioz, ahalbidetu daitekeenaren dimentsiora eramanez. Beharren arabera mapan lekutzeak aniztasunaren aitortza ahalbideratzen du, eta elkarrekin jardunean jartzen ditu; izan ere, funtsean, prozesu bat baita bestearen nortasunaren kontakizunerako sarbide izateko. Hori guztia azaldu nahi du artikulu honek, eta horren azalpenerako honako lau ardatzak hartzen dira kontuan: estruktura, epistemologia, metodologia eta fenomenologia.

\section{HITZ-GAKOAK:}

bitartekotasun, beharrak, kontakizun, zentsu, alteritate.
Para mediar adecuadamente, hace falta escuchar de verdad a los usuarios, no sólo como consumidores, sino también como productores; es necesario contar con medios y sistemas adecuados para escuchar y recoger la voz de los usuarios. El mapeo de necesidades nos ayuda a hacer esto. El mapeo de necesidades es tanto una metodología de investigación, como una intervención pedagógicaeducativa, un proceso que intenta poner de manifiesto la necesidad del sujeto, dándole forma, llevándola a la dimensión de lo real, a lo realizable, y por lo tanto, a la dimensión de lo posible. El mapeo de necesidades favorece también el reconocimiento de las diversidades, las pone en diálogo, porque, en esencia, se caracteriza por ser un proceso de introducción del otro en la narración de la identidad. Este artículo quiere explicar esto, siguiendo cuatro ejes expositivos: estructural, epistemológico, metodológico y fenomenológico.

\section{Palabras clave:}

mediación, necesidades, narración, sentido, alteridad. 


\section{Introducción}

Este artículo reflexiona sobre el papel del tercer sector como 'mediador social' entre los ciudadanos y las instituciones para conseguir una mayor cohesión social. La mediación representa un medio muy importante para 'hacerse cargo de...', porque activa procesos de mejor comprensión de uno mismo y de los contextos sociales, de crecimiento de las habilidades relacionales y, por lo tanto, del nivel de emancipación de los individuos. Dicho esto, traigo a este debate mi propuesta metodológica.

Para mediar adecuadamente, hace falta escuchar de verdad a los usuarios, no sólo como consumidores, sino también como productores; es necesario contar con medios y sistemas adecuados para escuchar y recoger la voz de los usuarios. Hay una distancia entre la demanda que viene de abajo (los usuarios), que no encuentra la manera de ser acogida y dirigida a los centros de decisión política y a los lugares de la planificación social, y los planes de acción que hace el tercer sector sobre la base de las directrices y objetivos establecidos en los niveles superiores. Mi propuesta se llama 'mapeo de necesidades': se trata de una metodología de investigación-acción compatible con una idea de tercer sector inspirada en una lógica del servicio que no está interesada principalmente en los sistemas de prestación de servicios, sino que se orienta a los usuarios, a las formas en que el usuario utiliza el servicio y al modo en que obtiene un valor de utilidad para su propia existencia, a través del uso del servicio.

Para llevar a cabo un servicio, siempre deberíamos preguntarnos qué es o no es apropiado en relación con el uso propuesto de los usuarios/beneficiarios para los cuales está diseñado. El mapeo de necesidades nos ayuda a hacer esto. El mapeo de necesidades es tanto una metodología de investigación, porque recoge las necesidades del usuario, las interpreta hermenéuticamente y las convierte en proyecto, como una intervención pedagógica-educativa, porque, gracias a la explicación de las necesidades, promueve un proceso de desarrollo de las capacidades estratégicas necesarias a los usuarios para traducir sus necesidades en el proyecto. El aumento de los niveles de la lectura y la planificación de los usuarios, alcanzado con el mapeo de necesidades, es una garantía de mayor eficacia de las acciones y servicios prestados por el tercer sector.

Voy a ilustrar estos temas siguiendo cuatro ejes expositivos: estructural, epistemológico, metodológico y fenomenológico.

\section{Eje estructural}

La necesidad es el asunto central de todas las acciones educativas y sociales. Investigar y comprender la necesidad es la primera etapa de toda intervención. Esta etapa no hay que verla como algo separado y autónomo de la intervención social, sino como intervención social; es ya una intervención social, un proceso de aprendizaje, cuyo objetivo es promover el cambio y el desarrollo en los sujetos que siguen este proceso.

El concepto de necesidad ha sido históricamente estructurado y desarrollado en las diferentes teorías y los distintos campos disciplinarios -economía, sociología, psicología-. Por esta razón, en la actualidad hay varias definiciones de necesidad. En general, hay una tendencia a considerarla en sentido negativo, es decir, como falta, carencia o ausencia; frente a esta concepción, está la que considera la necesidad como algo que se puede introducir con las intervenciones sociales y educativas, una respuesta que traza el camino que el sujeto debe completar. A mi juicio, esta definición es todavía insuficiente e inadecuada para proporcionar medios eficaces de acción dentro de la complejidad.

La complejidad de los sistemas sociales en que vivimos hoy día nos lleva a hacer referencia al enfoque de ecosistema, que intenta dar cuenta de la complejidad de los fenómenos naturales y la interrelación de los procesos humanos y de los actores/actrices, la naturaleza multifactorial de eventos y experiencias, las muchas miradas que pueden leer el mismo problema. Este marco teórico no admite, por supuesto, una visión reduccionista y mecanicista de la necesidad, ni propuestas para el diagnóstico ortopédico.

Mi propuesta es que es mucho más productivo, interesante y relevante desde el punto de vista pedagógico, desde una perspectiva de desarrollo, considerar la necesidad en un sentido positivo, es decir, como un recurso, subjetivamente significativo y relevante pedagógicamente: es un recurso que necesita ser investigado, interpretado, puesto en forma con el fin de llegar a un proyecto que es existencial, en primer lugar, y, por lo tanto, profesional y social. En este escenario, intervenciones educativas y de formación representan una palanca para que el sujeto pueda dar cuerpo al proyecto de desarrollo.

Es interesante empezar con la formulación teórica que Lévinas (1977) nos ofrece. Este autor propuso darle la vuelta a la visión tradicional negativa de la necesidad y sostuvo que la advertencia de la necesidad depende del diseño de la vida que podemos imaginar: la necesidad es la aspiración de vivir una situación de bienestar, el apetito de perfección, el deseo de plenitud. En este sentido, la necesidad es un recurso para el sujeto. Una vida feliz no es una vida sin necesidades, en la que el sujeto ha cumplido todas las necesidades; es importante tener la capacidad de fijarse de vez en cuando una meta para la propia existencia, es decir, tener la capacidad de re-imaginar una nueva necesidad por y para sí mismo.

La necesidad está relacionada con el proyecto. El proyecto es la manifestación de la intención constructiva del yo, que es perseguida por el sujeto a través de su larga experiencia vivida. Por esta razón, el proyecto se basa en la idea de 'presencia', combinada con la 
intención, con la que la persona se abre al mundo y trata de vivirlo. Desde esta tensión, nunca satisfecha, que es necesaria, surge la necesidad, que es el lugar de la elaboración activa del mundo, del objeto, del otro; es recurso que debe ser interrogado e interpretado para definir un proyecto capaz de articular en el tiempo, hacia un horizonte, la búsqueda de sentido que nos caracteriza como seres humanos, es decir, como seres narrativos, que trabajan para volver a interpretar, reducir, absorber la distancia/diferencia entre el yo y la alteridad, a través de una inversión en creatividad, imaginación y transformación de la realidad y de uno/a mismo/a.

Agnes Heller (1978), por su parte, dentro de una perspectiva marxista heterodoxa, señaló que la aspiración fundamental del ser humano, como ser socializado, es participar en la vida de una comunidad, en la que buscar la satisfacción de sus necesidades para el desarrollo. Por lo tanto, las necesidades son el resultado del equilibrio entre los impulsos identitarios individuales y las dimensiones relacionales, lo que guía y permite los caminos de la vida. En este sentido, creo que podemos hablar de la 'necesidad de comunidad'. La 'necesidad de comunidad' es la necesidad de cada persona de un espacio concreto en que las prácticas narrativas y discursivas son posibles para explorar la relación entre identidad y alteridad, la capacidad de socializar 'cara a cara', el encuentro de la mirada de otros, en un diálogo real entre los sujetos reales y concretos, y no entre los modelos ideales de los sujetos, la sociedad y la comunidad. La comunidad es el espacio de estas posibilidades. La necesidad de comunidad pone de manifiesto que la interpretación que cada persona hace de sí misma y del mundo tiene carácter social y cultural.

¿Cómo se puede satisfacer la 'necesidad de comunidad'? A esta pregunta, la lógica económica del capitalismo ha tratado, y trata, de ofrecer respuestas a través del mero consumo de cualquier bien, desde los bienes materiales hasta los bienes intangibles, que son el reservorio de símbolos y emociones, que están cada vez más comercializados y explotados por el sistema económico, como cualquier otro objeto. Sin embargo, el mero consumo propone sustitutos, es decir, simulacros aparentemente adecuados, pero, en realidad, completamente ineficaces, porque están vacíos; son un espejismo que promete, pero no mantiene, que simplemente alimenta el ciclo de la compulsión por consumir. La satisfacción de las necesidades no se puede reducir al acto de consumo (acción pasiva), sino que es, sobre todo, un acto de producción (acción activa). Es la productividad cultural y social, a la que cada individuo, inscrito dentro de los procesos de participación de la comunidad, contribuye. Cada individuo de la comunidad produce sus narraciones, y al hacerlo se enfrenta a las historias de los demás miembros de la comunidad, creando un intercambio de símbolos, valores, significados y prácticas (Manfreda, 2010a).

En términos más generales, podemos decir que las necesidades de los sujetos son un producto de nuestra necesidad de 'ser' en el mundo en continua búsqueda de significado.

\section{Eje epistemológico}

Las ciencias humanas, hoy más que nunca, se enfrentan con la categoría del cuidado. Las teorías de estas ciencias eligen las prácticas que cada vez utilizan más los métodos de 'hacerse cargo de' para tratar de activar los procesos de cambio y desarrollo (Cambi, 2010). El acto de cuidar no sólo es una intervención en favor de una persona débil, enferma, que necesita ayuda, sino también un medio para la emancipación de los sujetos, para que puedan desarrollar su potencial. Educar significa hacerse cargo del sujeto, del grupo, de una entidad social, mediante la comprensión y la planificación, a través del acto de donación y de dedicación, junto con la empatía y la razón. Cuidar a alguien es asumir la carga de su crecimiento, que se lleva a cabo en la autonomía del sujeto o sujetos que se encuentran en la educación, pero debe ser guiado (Cambi, 2010).

Hasta ahora hemos dicho que el cuidado tiene que ver con la emancipación, pero también significa participación, adhesión, empatía; cuidado es la búsqueda de sentido dentro del proceso abierto y continuo que es la identidad, dentro de la re-construcción del yo, siempre problemática. Mi idea de la necesidad parte de este marco teórico, y se dirige a la pedagogía social y a la pedagogía de la comunidad para obtener las bases científicas de una metodología de investigación coherente. La referencia a la pedagogía de comunidad me lleva a sostener que la necesidad está muy contextualizada desde el punto de vista cultural, social y comunitario. Creo también que es útil combinar la necesidad, su investigación y comprensión, con las representaciones sociales compartidas en el contexto estudiado. La teoría de las representaciones sociales intenta articular la relación entre los factores individuales y los factores sociales en la construcción de representaciones, que median las respuestas conductuales de los sujetos, frente a los problemas relacionados con su vida cotidiana. Esta teoría destaca la "esencia social y colectiva de la comprensión que las personas tienen de sí mismas y del mundo, centrándose en ideas compartidas, en la manera en que se desarrollan, se describen, se modifican" (Zani y Cicognani, 2000). Las representaciones sociales son una forma de conocimiento práctico, el desarrollo de un objeto social por parte de una comunidad que permite a sus miembros comportarse y comunicarse de una manera comprensible.

Esquemáticamente, podemos decir que las representaciones sociales son:

\section{- Una forma de conocer.}

- Un sistema de valores, significados y prácticas que organizan la realidad, permitiendo a quienes los comparten orientarse en su contexto de referencia material y cultural, y actuar. 
- Una lista de categorías con las que empezar a dar sentido a lo que es nuevo.

Las representaciones sociales orientan el comportamiento y las prácticas de los individuos en sus interacciones sociales, debido a que:

- Construyen y dan forma a la realidad.

- Median en la comunicación y la interacción social.

- Definen los límites de un grupo.

- Hacen que lo desconocido se vuelva conocido.

La formulación de las necesidades se lleva a cabo en el marco de las representaciones. Éstas son tanto el fondo - lo que afecta al sujeto, en la formulación de sus necesidades- como un conjunto de claves para la interpretación que el investigador debe tomar necesariamente en consideración, en el proceso de exploración de las necesidades de un determinado grupo social.

\section{Eje metodológico}

¿Cómo investigar la necesidad? El análisis de las necesidades, en general, se configura como una investigación dirigida a recopilar datos e información útil para el diseño de intervenciones educativas y formativas. Es en el ámbito de la formación donde más se ha reflexionado sobre el análisis de las necesidades, las metodologías que deben utilizarse, los propósitos y los objetivos que deben alcanzarse. En materia de educación, sin embargo, el análisis de las necesidades no ha sido suficientemente problematizado para reflexionar sobre las características específicas que debe tener de forma que pueda ser utilizado con éxito en intervenciones socioeducativas. ¿Cuáles son los métodos más adecuados? ¿Cuáles son los fines a los que dar respuesta? ¿Cuál es su significado? En situación operativa, las reflexiones maduradas en el campo de la formación han sido directamente traducidas y adaptadas al ámbito socioeducativo. Así pues, considero que las necesidades y su detección en el contexto socioeducativo merecen una consideración especial.

Existen algunas diferencias fundamentales entre los ámbitos formativo y socioeducativo:

- De contexto:

- En el caso de la formación, la intervención se diseña para el contexto socioeconómico, organizativo y de trabajo, sobre la base del análisis de las necesidades.

- En el caso de las acciones socioeducativas, el contexto es social, cultural, relacional, ambiental; dentro de ese contexto, se coloca la vida de sus miembros y, en particular, de quienes, por quienes y con quienes queremos crear una intervención educativa.
- De objetivo:

- En el caso de la formación, el objetivo se traza principalmente para definir el perfil de las competencias que posee el sujeto y cuáles deben ser desarrolladas a través de un curso de formación.

- En el caso de las acciones socioeducativas, el objetivo es principalmente de asesoramiento: se busca prestar apoyo para que el sujeto pueda hacer un proceso de reflexión sobre sí mismo, valorizar su especificidad, leer sus contextos de vida de la mejor manera, y así, promover o mejorar sus condiciones de emancipación.

Por otra parte, en la formación, podemos resumir las principales áreas y los objetos del análisis de las necesidades, en dos grandes categorías:

- Análisis de las necesidades de la organización: se refiere a todos los actores/actrices de la organización, y su objetivo es superar los problemas, promover el cambio organizacional, identificar planes de formación y puesta al día de los recursos humanos.

- Análisis de las necesidades de los individuos: está diseñado para la orientación profesional, o para proporcionar a las instituciones formativas los datos necesarios para definir y diseñar programas de formación para la profesión y el trabajo (en este caso, la atención se centra principalmente en las competencias técnicas y profesionales de la persona, y por lo tanto, el análisis de necesidades está muy cerca del balance de competencias).

¿Qué pasa en el contexto socioeducativo? Sucede que las dos dimensiones que se han indicado anteriormente asumen características diferentes, y por lo tanto, requieren de un marco teórico y metodológico muy específico:

- Ya no hablamos de organizaciones, sino de grupos y comunidades.

- La investigación de las necesidades individuales no se centra en las competencias técnicas y profesionales (que son importantes, pero en una escala diferente), sino en las competencias emocionales y relacionales, contextuales, y en todas las dimensiones que se combinan para definir una condición de emancipación individual, y por lo tanto, de capacidad de establecer por sí mismo/a un proyecto de vida.

Veamos las consecuencias metodológicas que se derivan de esas dimensiones. En primer lugar, la metodología escogida, debe tener características predominantemente cualitativas, etnográficas y, sobre todo, indiciarias. No anhela resultados exhaustivos, definitivos ni de definición. Esto claramente implica un alto grado de incertidumbre, que debe tenerse en cuenta, pero al mismo tiempo, el necesa- 
rio grado de apertura y flexibilidad, lo que garantiza más posibilidades de intervención:

- En un profundo respeto por la diversidad.

- Lo más libre posible de los estereotipos y prejuicios.

- De acuerdo con una actitud que valoriza realmente al otro.

- De manera coherente con el marco de significado, representado por el contexto de vida en el que los actores/actrices están inmersos.

Debido a todas estas consideraciones, mi propuesta metodológica se llama mapeo de necesidades. El mapeo de necesidades no es un proceso dirigido a encontrar algo que ya existe, sino un proceso para tratar de poner de manifiesto la necesidad del sujeto, dándole forma, llevándola a la dimensión de lo real, a lo realizable, y por lo tanto, a la dimensión de lo posible. Conducir la necesidad a la dimensión de lo real significa hacer progresar la necesidad en un proyecto. Es decir, introducir el espacio de reflexión para hacer explícito lo que, hasta ese momento, se incorpora y está implícito en las acciones del sujeto. El mapeo se coloca entre la necesidad y el proyecto, y trata de facilitar y simplificar la transición de una a otro. Ésta es una actividad que investiga las necesidades, no como si fueran objetos, que se recogen directamente en las personas y son directamente inteligibles para ellas. Es, más bien, un proceso interpretativo, de comprensión y de co-construcción de las necesidades, las cuales se componen en una narrativa posible y socialmente integrada.

El mapeo de necesidades, por lo tanto, tiene una impronta fenomenológica, es abierto, interactivo, y toma la postura metodológica de la observación y de la escucha. Tiene además caracteres ideográficos, porque quiere comprender las estrategias a través de las cuales los individuos construyen significados, para aumentar el nivel de conciencia y sentar las bases para la promoción de la participación, la capacidad de cambio de diseño, y por lo tanto, la emancipación individual y comunitaria (Colazzo 2008). El desafío del mapeo de necesidades es:

integrar los datos estructurales y las variables subjetivas, como las representaciones sociales, los modelos simbólicos y culturales, las expectativas hacia el futuro, las experiencias personales, porque sólo si todos ellos están relacionados, puede encontrar los significados que caracterizan un determinado contexto (Navanco y Novara, 2006).

En este contexto, el investigador se configura como un agente de cambio.

El término 'mapeo', utilizado en vez de 'análisis', da mejor cuenta que este último término de una obra que es, ante todo, un recorrido de descubrimiento de huellas, de elementos de pequeño tamaño, que termina componiendo una imagen representativa, donde adquiere gran importancia la co-construcción hermenéutica, de lecturas subjetivas y objetivas, situadas, particulares, atentas al 'aquí y ahora' de todos los actores/actrices involucrados/as en el proceso. Utilizar el término 'mapeo', nos permite mantener bien claro el carácter representativo, y de interpretación, de la investigación de/sobre las necesidades. Es el resultado de un espacio de trabajo compartido, que siempre puede ser revisado, modificado y rediseñado, porque es intrínsecamente abierto. Mi propuesta de mapeo de necesidades asume el carácter de una actividad de asesoramiento cuyo objetivo es fomentar, en el sujeto, un trabajo de remodelación activa del mundo, para poder hacer evolucionar la necesidad en un proyecto. El hecho de que hablamos de trabajo indica que es un proceso que requiere esfuerzo, compromiso, responsabilidad; un proceso que se desarrolla en el tiempo e involucra a la memoria. También indica que es una tarea que cada sujeto tiene que dirigir hacia sí mismo, por sí mismo, por y para su emancipación. El mapeo de necesidades debe ser considerado como una actividad de investigación educativa, para facilitar, en el contexto de los actores/actrices, el reconocimiento de sus necesidades y su articulación en el proyecto.

El proyecto debe estar vinculado necesariamente a una intrínseca apertura, que se caracteriza por:

- Una indeterminación a priori de los resultados.

- Un sentido procesual.

- Dinamismo.

- Complejidad.

El acuerdo con el sentido de apertura no justifica el abandono del proyecto, no niega y ni frustra el proyecto, no le quita valor, como tampoco significado. Todo lo contrario. El proyecto es verdaderamente un proyecto si es capaz de conjugar, al mismo tiempo, la intencionalidad y la apertura. El mapeo de necesidades promueve la definición de un proyecto para el desarrollo personal, en el contexto del cual se forma parte, visto como una interpretación creativa de la existencia, pero basada en objetivos al alcance, para estar siempre en diálogo con los demás y la realidad, sin caer en los extremos de:

- La mera reproducción de conformismo social.

- Un proyecto, poco realista, no contextual, y por lo tanto, fantasmal, $y$, a veces, incluso delirante.

El mapeo de necesidades es un proceso de suspensión de la vida cotidiana, a fin de establecer un lugar de encuentro entre la imaginación, la creatividad, la productividad y el proyecto. Es decir, un espacio adecuado para formular una representación de las necesidades. Además, el mapeo de necesidades considera el sujeto, en su ser inscrito en contextos específicos de la vida, dentro de los procesos específicos de comunicación y de relación entre determinados grupos y comunidades de práctica. Es por eso 
por lo que tratamos de incluir, en el acto en el que se realiza el mapeo, el sistema de relaciones sociales del sujeto, los otros actores/actrices del contexto, que, por diversas razones, son parte de su constelación relacional, y el marco de sentido en el que las prácticas discursivas y el procesamiento de los significados son realizados por el sujeto. Por estas razones, el mapeo de necesidades es metodológicamente una investigación-intervención.

Los métodos de intervención del mapeo de necesidades consisten en una función narrativa, en la que la interacción entre el sujeto y el investigador es fundamental. Las narraciones son el resultado

\begin{abstract}
"de la construcción de un escenario, complejo y coherente, por el sujeto que narra, integrado por los acontecimientos, que están inseparablemente relacionados con factores emocionales, las creencias personales, las preferencias, el conocimiento científico, las decisiones, las acciones, y así sucesivamente. Esta construcción está implícita en la interpretación, implícita o explícita, consciente o inconsciente, de lo que sucedió. El mismo conjunto de eventos, se puede ordenar temporalmente, y estructurar lógicamente, de diferentes maneras por diferentes narradores, que participan de manera directa, o indirecta, en los eventos" (Giani, 2009).
\end{abstract}

La narración es la forma específica (el proceso cognitivo específico, escribió Bruner [2001]), a través de la cual los seres humanos buscan y dan forma y sentido a las cosas, a sus experiencias cotidianas, a su propia existencia, al contexto en el que viven. Existe una estrecha relación entre narrarse a sí mismo y formarse. A medida que el sujeto narra, produce una reconstrucción significativa de su propia vivencia, recruzando y reorganizando, dando sentido a los acontecimientos y situaciones de la historia personal.

Sin embargo, el objetivo de la función narrativa del mapeo de necesidades es acompañar al sujeto, para pasar de 'narrar', hacia un 're-narrar'. Esto significa hacer que aparezca la alteridad dentro de la narración, romper la unidad de la narración en primera persona, y por lo tanto, producir un desplazamiento. La ruptura es funcional, sirve para producir un proceso de re-significación de sí mismo y la realidad circunstante.

El mapeo de necesidades, utiliza las técnicas de interacción dialógica, ya que representan un dispositivo dinámico, que mantiene unidos a los procesos de diferenciación y de procesos de integración. El diálogo con la alteridad -en mi opinión- puede llevarse a cabo a través de dos movimientos posibles:

- Saliendo de sí mismo, para tratar de estar de parte del otro, desde su punto de vista. Se trata de juzgar a otra persona, asumir otra identidad, de manera temporal, para enfrentarse a otra mirada, para tratar de comprender a los otros, pero, sobre todo, para comprender nuestra mirada.
- Buscando dentro de nosotros mismos, esa parte de 'no yo' que habita en nosotros, el otro que podemos reconocer en nosotros.

Esto crea un efecto de espejo que permite ampliar los límites del sentido y avanzar hacia escenarios más posibles, teniendo en cuenta el proyecto. En estas condiciones, puede ocurrir un cambio productivo. Bajo estas condiciones, el mapeo de necesidades se puede definir como investigación-intervención.

\section{Eje fenomenológico}

La cohesión social es un concepto compuesto. Tiene que ver con la calidad de vida, con las condiciones de empleo, con la salud y el bienestar, con la lucha contra la pobreza y la exclusión social. Por esta razón, es un objetivo que se logra no sólo mediante el aumento de las redes de relaciones interpersonales de desarrollo económico y de mercado. Es necesario, intervenir en el desarrollo y fortalecimiento de los lazos sociales, porque tienen un papel fundamental para el crecimiento de los grupos y comunidades, para el bienestar social, para la calidad de vida en general (Ingrosso, 2006). Es, por tanto, un concepto y un valor que no puede reducirse a mera coexistencia pacífica: un proceso de intercambio, convergencia de intereses y proyectos, mantenimiento y desarrollo de capital social y redes comunitarias.

Los factores que actúan en la dirección favorable a la consecución de la cohesión social son:

- La territorialización de los servicios públicos de infraestructura y recursos.

- El reparto de responsabilidades entre las autoridades del orden público y las administraciones locales, dentro de un concepto amplio de seguridad, que también incluye la habitabilidad social, la calidad de vida, de trabajo y el ocio.

- El aumento de los espacios físicos y de acción social para el sector del voluntariado y el tercer sector.

- El aumento de las situaciones que requieren más responsabilidad individual y la integración de los servicios públicos (Ingrosso, 2006).

Cuando no hay cohesión social, o está en crisis, es esencial la mediación social por parte los actores del tercer sector. De hecho, la mediación:

incluye las prácticas de intervención dirigidas a la reconstrucción de los lazos sociales con el fin de re-socializar a los individuos, y para luchar contra los fenómenos de la desorganización, que atentan contra la vida colectiva.

Es una forma de intervención, que tiene especial importancia para todas las situaciones límite de los individuos, o grupos, que mantienen, con dificultad, vínculos positivos con sus contextos 
de pertenencia, o el contexto social más amplio (Bramanti, 2005).

Tiene que ser un medio de conexión entre los diferentes sujetos, que están buscando fórmulas de convivencia, y debe ser capaz de interpretar estas diferencias como un enriquecimiento recíproco. Esto es absolutamente necesario en una sociedad, que es algo orgánico, pero, al mismo tiempo, también una convivencia de comunidades, micro y macroculturas, diferentes personas, cada una caracterizada por una cultura local, a menudo irreconciliables; una convivencia que es posible, precisamente, porque hay un marco, que lo hace posible.

En este modelo de servicios sociales 'orientados al cliente' al que me refiero, cada sujeto tiene un potencial para el desarrollo que se realiza si se crean las condiciones, que pueden ser la ayuda y el apoyo por parte una persona más competente, de manera que la distancia entre el nivel de desarrollo actual y el nivel de desarrollo potencial pueda llenarse. La mediación es, ante todo, una acción de escuchar y está orientada al reconocimiento de las necesidades de las personas, a fin de facilitar su conexión, su comparación, sus intercambios, su composición productiva; no obstante todo también creíble, dentro de los escenarios contextuales de referencia. El acto de mediación es una composición que viene a través de la escucha, y al reconocimiento de la diversidad y la reciprocidad en la diversidad. La mediación social apunta a la prevención y a la gestión positiva de conflictos socioeconómicos, culturales, étnicos, a través de la restauración de las conexiones y las relaciones sociales, y del desarrollo de competencias de los individuos y grupos.

El mapeo de necesidades puede ser un instrumento importante para la mediación social, a través del cual se pueden promover nuevas reglas de comunicación e interacción entre los ciudadanos/ciudadanas y las instituciones. El mapeo de necesidades tiene la ambición de combinar la diversidad y la cohesión social. Ambas son compatibles, en mi opinión, aunque esto puede parecer una contradicción. La premisa fundamental de la cohesión social no es 'ser iguales', sino 'reconocerse unos a otros, en la diversidad' (porque siempre es diferente uno de otro) y 'estar en diálogo'. El mapeo de necesidades favorece el reconocimiento de las diversidades, las pone en diálogo, porque, en esencia, se caracteriza por ser un proceso de introducción del otro en la narración de la identidad. Sólo de esta manera puede completarse el camino del autoconocimiento. La identidad se define únicamente en su encuentro con la alteridad y depende fundamentalmente de la relación establecida con ella. La diferencia es constitutiva del ser, del yo, de su siempre cambiante dinámica, y por lo tanto, no se puede borrar, marginar, eliminar o cancelar sin afectar negativamente a la misma identidad.

Para descubrir y definir el yo, hay que pasar a través de la diversidad, hay que interactuar con el otro, diferente del yo: el yo, de esta manera, se define en su encuentro con el otro. La identidad no es un dato definitivo e inmutable, no es un sistema cerrado, una fortaleza, que debe ser defendida contra amenazas externas. No es algo ontológico, un a priori monolítico, sino una entidad dinámica, semipermeable, y por lo tanto, contingente, histórica y procesal, en continuo diálogo con el contexto en el que está inmerso el sujeto.

En esta dinámica, es de gran importancia el término 'reconocimiento'; reconocimiento mutuo, sin duda, pero sobre todo, reconocimiento del otro, como condición para ser capaz de reconocerse a sí mismo, como un requisito necesario en el proceso de definición de la identidad. Sin embargo, la existencia del otro en nosotros requiere otra acción fundamental: la acogida. Es decir, la presencia de la alteridad en el yo, el otro en nosotros, sin que ello signifique el deterioro del sujeto que lo acoge. Acogida y reconocimiento son dos palabras clave en el proceso de definición de la identidad. El otro representa "la forma misma que toma el sí, donde el interior se cruza con el mundo exterior, el suyo con el extraño" (Esposito, 2002: 207). Esto no quiere decir que el exterior no exista, que el otro no exista; quiere decir que 'dentro' y 'fuera' se derivan del proceso continuo de su comparación, intercambio y relación que los determina como tal, en una dinámica de co-existencia, que llega a un 'externo internalizado' y una 'interna exteriorizada', en el que la identidad es el resultado de su producto.

Los procesos de desarrollo y emancipación individual y social, son, por lo tanto, siempre, procesos de comparación, procesamiento, constante re-significación de la alteridad, de cualquier forma. Por lo tanto, se deduce que el mapeo de necesidades es particularmente importante para todos los proyectos socioeducativos que se refieren a la interculturalidad y la integración, en los que el destinatario es el marginal, el extraño. 


\section{Referencias bibliográficas}

BRAMANTI, D. (2005): Sociologia della mediazione. Teorie e pratiche della mediazione di comunità, Milán, Franco Angeli.

BRUNER, J. (2001): “L'interpretazione narrativa della realtà", en BRUNER, J., La cultura dell'educazione. Nuovi orizzonti per la scuola, Milán, Feltrinelli.

CAMBI, F. (2010): La cura di sé come processo formativo. Tra adultità e scuola, Roma, Laterza.

COLAZZO, S. (2008): Progettazione e valutazione dell'intervento formativo, Milán, McGraw Hill.

ESPOSITO, R. (2002): Immunitas. Protezione e negazione della vita, Turín, Einaudi.

GIANI, U. (2009): “Le traiettorie del malessere: modello multi metodologico per l'analisi delle narrative di malattia”, en GIANI, U. (ed.), Narrative based medicine e complessità, Nápoles, Scriptaweb, págs. 47-78.

HELLER, A. (1978): La teoria dei bisogni in Marx, Milán, Feltrinelli.

INGROSSO, M. (ed.) [2006]: La promozione del benessere sociale: progetti e politiche nelle comunità locali, Milán, Franco Angeli.
LAVANCO, G.; y NOVARA, C. (2006): Elementi di psicologia di comunità. Dalla teoria all'intervento, Milán, McGraw Hill.

LÉVINAS, E. (1977): Totalità e infinito. Saggio sull'esteriorità, Milán, JacaBook.

MANFREDA, A. (2011): "Narrazione e mappatura dei bisogni di salute", en MINELLI, M.; PERUCCA, A.; y SIMONE, M. G. (eds.), Una nuova cultura per la pratica medica, Lecce, Edizioni di Solidarietà, págs. 45-62.

- (2010a): “'Laboratorio memoria': ricerca educativa e bisogno di comunità", en COLAZZO, S. (ed.), Sapere pedagogico. Scritti in onore di Nicola Paparella, Roma, Armando, págs. 674-683.

- (2010b): “Una metafora influente: l'io immunitario. Dalla pedagogia della salute alla pedagogia di comunita", en In medias res. Studia resoviensia in lingua et litteris, Rzeszów, Wydawnictwo Promar-International, págs. 125-143.

ZANI, B.; y CICOGNANI, E. (2000): Psicologia della salute, Bolonia, Il Mulino. 